\title{
Phase II Trial of Cediranib in Combination With Cisplatin and Pemetrexed in Chemotherapy-Naïve Patients With Unresectable Malignant Pleural Mesothelioma (SWOG S0905)
}

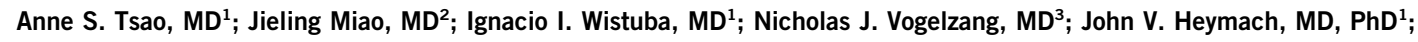

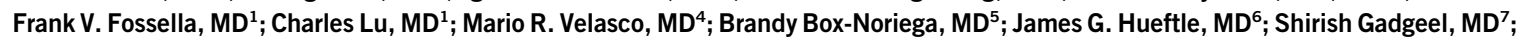
Mary W. Redman, $\mathrm{PhD}^{2}$; David R. Gandara, $\mathrm{MD}^{8}$; and Karen Kelly, MD ${ }^{8}$ on behalf of the SWOG investigators.

PURPOSE Antiangiogenic agents combined with chemotherapy have efficacy in the treatment of unresectable malignant pleural mesothelioma (MPM). Cediranib (AstraZeneca, Cheshire, United Kingdom), a vascular endothelial growth factor receptor and platelet-derived growth factor receptor inhibitor, demonstrated therapeutic potential in a prior phase I trial. We evaluated a phase II trial for efficacy.

PATIENTS AND METHODS SWOG S0905 (ClinicalTrials.gov identifier: NCT01064648) randomly assigned cediranib or placebo with platinum-pemetrexed for six cycles followed by maintenance cediranib or placebo in unresectable chemotherapy-naive patients with MPM of any histologic subtype. Primary end point was Response Evaluation Criteria in Solid Tumors (RECIST) version 1.1 progression-free survival (PFS). Secondary end points included overall survival, PFS by modified RECIST v1.1, response (modified RECIST and RECIST V1.1), disease control, and safety/toxicity. The trial was designed to detect a difference in RECIST v1.1 PFS at the onesided 0.1 level using a stratified log-rank test.

RESULTS Ninety-two eligible patients were enrolled (75\% epithelioid and 25\% biphasic or sarcomatoid). The cediranib arm had more grade 3 and 4 diarrhea, dehydration, hypertension, and weight loss. Cediranib improved PFS by RECIST v1.1 (hazard ratio, $0.71 ; 80 \% \mathrm{Cl}, 0.54$ to $0.95 ; P=.062 ; 7.2$ months $v 5.6$ months) and increased modified RECIST v1.1 response (50\% v 20\%; $P=.006$ ). By modified RECIST v1.1, cediranib numerically increased PFS (hazard ratio, $0.77 ; 80 \% \mathrm{Cl}, 0.59$ to $1.02 ; P=.12$; median, 6.9 months $\vee 5.6$

ASSOCIATED

CONTENT

Appendix

Data Supplement

Author affiliations

and support

information (if

applicable) appear

at the end of this

article.

Accepted on July 9, 2019 and published at jco.org on August 6, 2019: DOI https://doi. org/10.1200/JC0.19. 00269

The content is solely the responsibility of the authors and does not necessarily

represent the official views of the National Institutes of Health.

Clinical trial

information:

NCT01064648. months). No significant difference in overall survival was observed.

CONCLUSION The addition of cediranib to platinum-pemetrexed improved PFS by RECIST V1.1 and response rate by modified RECIST in patients with unresectable MPM. Whereas adding antiangiogenics to chemotherapy has been a successful strategy for some patients, the cediranib toxicity profile and small incremental survival benefit precludes additional development in MPM.

J Clin Oncol 37:2537-2547. @ 2019 by American Society of Clinical Oncology

\section{INTRODUCTION}

Malignant pleural mesothelioma (MPM) is a rare disease with few treatment options and poor survival outcomes. ${ }^{1}$ Identifying targets for therapy is challenging. Mesothelioma secretes high levels of vascular endothelial growth factor (VEGF) and utilizes the platelet-derived growth factor and receptor (PDGF/ PDGFR) pathway for autocrine growth stimulation. ${ }^{2-4}$ PDGFR inhibition increases chemotherapy uptake into tumor cells via pericyte manipulation and decreased interstitial fluid pressure. ${ }^{5,6}$ These factors suggest that the angiogenic pathway is clinically relevant and that targeting both VEGF and PDGF with systemic chemotherapy may be beneficial.
In 2003, cisplatin-pemetrexed received regulatory approval for use in the front-line setting ${ }^{1}$ and, to date, remains a standard of care worldwide. However, in 2016, the phase II and III Mesothelioma Avastin Cisplatin Pemetrexed Study (MAPS; ClinicalTrials. gov identifier: NCT00651456) ${ }^{7}$ demonstrated that bevacizumab combined with cisplatin-pemetrexed increased survival outcomes. ${ }^{7}$ In addition, in 2017, the phase II LUME-Meso trial (ClinicalTrials.gov identifier: NCT01907100) demonstrated a progressionfree survival (PFS) benefit with the addition of nintedanib, a VEGF receptor (VEGFR) inhibitor, to cisplatin-pemetrexed. ${ }^{8}$ However, in September 2018, the subsequent phase III LUME-Meso trial was negative for a PFS benefit. ${ }^{9}$ Antiangiogenics have not received 


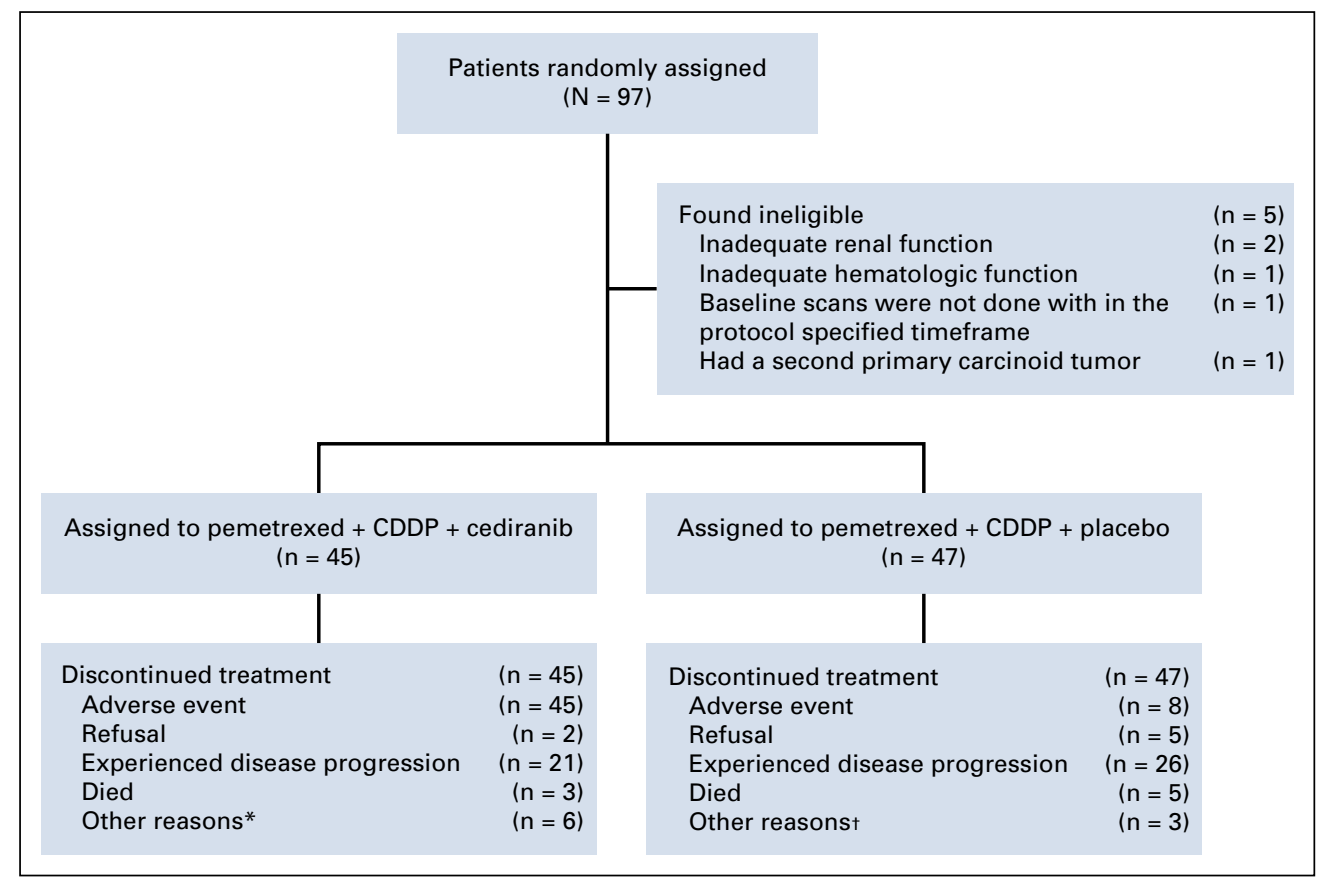

FIG 1. CONSORT diagram. (*) Patients' offtreatment notice indicated off because of disease progression, but additional documentation of progression is needed; $(n=4)$; physician decision $(n=1)$; treatment delay greater than protocol defined $(n=1) .(\dagger)$ Surgical intervention $(n=1)$; physician decision $(n=1)$; patient's off-treatment notice indicated off because of disease progression, but additional documentation of progression is needed. $(n=1)$.

agency approval yet, but bevacizumab is commonly administered in addition to platinum-pemetrexed in the frontline setting.

Cediranib (AstraZeneca, Cheshire, United Kingdom), an oral tyrosine kinase inhibitor of VEGFR $1,2,3$ and PDGFR has been evaluated in mesothelioma as monotherapy and in combination with chemotherapy. Southwest Oncology Group trial SWOG 0509 reported a 9\% response rate and $34 \%$ disease stabilization with cediranib in patients with previously treated mesothelioma. ${ }^{10}$ We theorized that targeting VEGFR and PDGFR would enhance chemotherapy efficacy and potentially bypass resistance mechanisms of high VEGF ligand secretion. ${ }^{11}$ SWOG 0905 was thus designed as a phase I and II front-line trial. The phase I trial established the maximum tolerated dose of cediranib at $20 \mathrm{mg}$ per day when combined with cisplatin-pemetrexed..$^{12}$ Median RECIST v1.1 PFS was 12.8 months, median modified RECIST (mRECIST) PFS was 8.6 months, and median overall survival (OS) was 16.2 months. ${ }^{12}$ Here, we report the results of the randomized phase II SWOG 0905 trial.

\section{PATIENTS AND METHODS}

Key inclusion criteria included any histology MPM, measurable or nonmeasurable disease, Zubrod performance status (PS) equal or less than 2 , and adequate organ function. Prior surgery or prior radiation therapy was allowed. No prior systemic therapy (chemotherapy or biologic) was allowed, unless it was neoadjuvant chemotherapy as part of definitive treatment ( $\geq 6$ months before enrollment). Patients were noted as having a prior surgery if the procedure was for definitive intent (ie, pleurectomy/ decortication or extrapleural pneumonectomy). Exclusion criteria included severe systemic comorbid disease, significant cardiac history, uncontrolled hypertension, significant proteinuria, prolonged QTc interval, history of hemoptysis, bleeding diathesis, or an inability to take oral medication.

The protocol and informed consent document were approved by the National Cancer Institute and the SWOG member sites' institutional review boards. This study was monitored by the SWOG Data and Safety Monitoring Committee.

Radiographic imaging was performed after every two treatment cycles, and tumor measurements were determined by both RECIST v1.1 and mRECIST. ${ }^{13,14}$ mRECIST measurements rely on measuring tumor thickness perpendicular to the chest wall or mediastinum in two positions at three separate levels on computed tomography scans. These six measurements are summed into a pleural unidimensional measure. Any lymph nodes or bidimensionally measurable lesions are measured unidimensionally by RECIST. All measurements are added to obtain the total tumor measurement. Cisplatin $\left(75 \mathrm{mg} / \mathrm{m}^{2}\right)$ plus pemetrexed $\left(500 \mathrm{mg} / \mathrm{m}^{2}\right)$ was administered intravenously every 3 weeks with dose modifications conducted by standard guidelines. Carboplatin substitution was allowed if the patient developed toxicity to cisplatin. Cediranib $20 \mathrm{mg}$ per day or placebo was administered concomitantly on day 1 and continued daily.

\section{Random Assignment}

Patients were randomly assigned with equal probability (1:1) between cisplatin-pemetrexed with cediranib versus placebo. Randomization was stratified by PS (0 to $1 v 2$ ) 
TABLE 1. Patient Demographics

\begin{tabular}{|c|c|c|c|}
\hline Patient Demographic & $\begin{array}{c}\text { Total } \\
(\mathrm{N}=92)\end{array}$ & $\begin{array}{l}\text { Platinum + Pemetrexed } \\
+ \text { Cediranib }(n=45)\end{array}$ & $\begin{array}{l}\text { Platinum + Pemetrexed } \\
+ \text { Placebo }(n=47)\end{array}$ \\
\hline \multicolumn{4}{|l|}{ Age, years } \\
\hline Median & 72 & 72 & 72 \\
\hline Range & $(46-85)$ & $(46-82)$ & $(51-85)$ \\
\hline \multicolumn{4}{|l|}{ Sex } \\
\hline Male & $78(85)$ & $38(84)$ & $40(85)$ \\
\hline Female & $14(15)$ & $7(16)$ & $7(15)$ \\
\hline \multicolumn{4}{|l|}{ Ethnicity } \\
\hline White & 85 (93) & $43(96)$ & $42(89)$ \\
\hline African American & $4(4)$ & $1(2)$ & $3(6)$ \\
\hline Unknown & $3(3)$ & $1(2)$ & $2(4)$ \\
\hline \multicolumn{4}{|l|}{ Hispanic } \\
\hline Yes & $5(5)$ & $3(7)$ & $2(4)$ \\
\hline No & $85(92)$ & $41(91)$ & $44(94)$ \\
\hline Unknown & $2(2)$ & $1(2)$ & $1(2)$ \\
\hline \multicolumn{4}{|l|}{ Zubrod PS } \\
\hline $0-1$ & $86(93)$ & $42(93)$ & $44(94)$ \\
\hline 2 & $6(7)$ & $3(7)$ & $3(6)$ \\
\hline \multicolumn{4}{|l|}{ Histology } \\
\hline Epithelioid & $69(75)$ & $34(76)$ & $35(74)$ \\
\hline Biphasic or sarcomatoid & $23(25)$ & $11(24)$ & $12(26)$ \\
\hline \multicolumn{4}{|l|}{ Definitive surgery } \\
\hline Yes & $18(20)$ & $12(27)$ & $6(13)$ \\
\hline Right partial P/D & $3(17)$ & $2(17)$ & $1(17)$ \\
\hline Right P/D & $4(22)$ & $2(17)$ & $2(33)$ \\
\hline Left partial P/D & $2(11)$ & $2(17)$ & $0(0)$ \\
\hline Left P/D & $3(17)$ & $3(25)$ & $0(0)$ \\
\hline Right EPP & $3(17)$ & $1(8)$ & $2(33)$ \\
\hline Left EPP & $3(17)$ & $2(17)$ & $1(17)$ \\
\hline No surgery & $74(80)$ & $33(73)$ & $41(87)$ \\
\hline \multicolumn{4}{|l|}{ Prior history of palliative RT } \\
\hline Yes & $17(18)$ & $8(18)$ & $9(19)$ \\
\hline No & $75(82)$ & $37(82)$ & $38(81)$ \\
\hline \multicolumn{4}{|c|}{ Prior history of chemotherapy as neoadjuvant/adjuvant } \\
\hline Yes & $12(13)$ & $6(13)$ & $6(13)$ \\
\hline No & $80(87)$ & $39(87)$ & $41(87)$ \\
\hline \multicolumn{4}{|l|}{ Baseline RECIST v1.1 } \\
\hline Measurable & $75(82)$ & $35(78)$ & $40(85)$ \\
\hline Nonmeasurable & $17(18)$ & $10(22)$ & $7(15)$ \\
\hline \multicolumn{4}{|c|}{ Baseline modified RECIST v1.1 } \\
\hline Measurable & $66(72)$ & $36(80)$ & $30(64)$ \\
\hline Nonmeasurable & $26(28)$ & $9(20)$ & $17(36)$ \\
\hline
\end{tabular}

NOTE. Data are given as No. (\%), unless otherwise noted.

Abbreviations: EPP, extrapleural pneumonectomy; P/D, pleurectomy decortication; PS, performance status; RT, radiation therapy. 


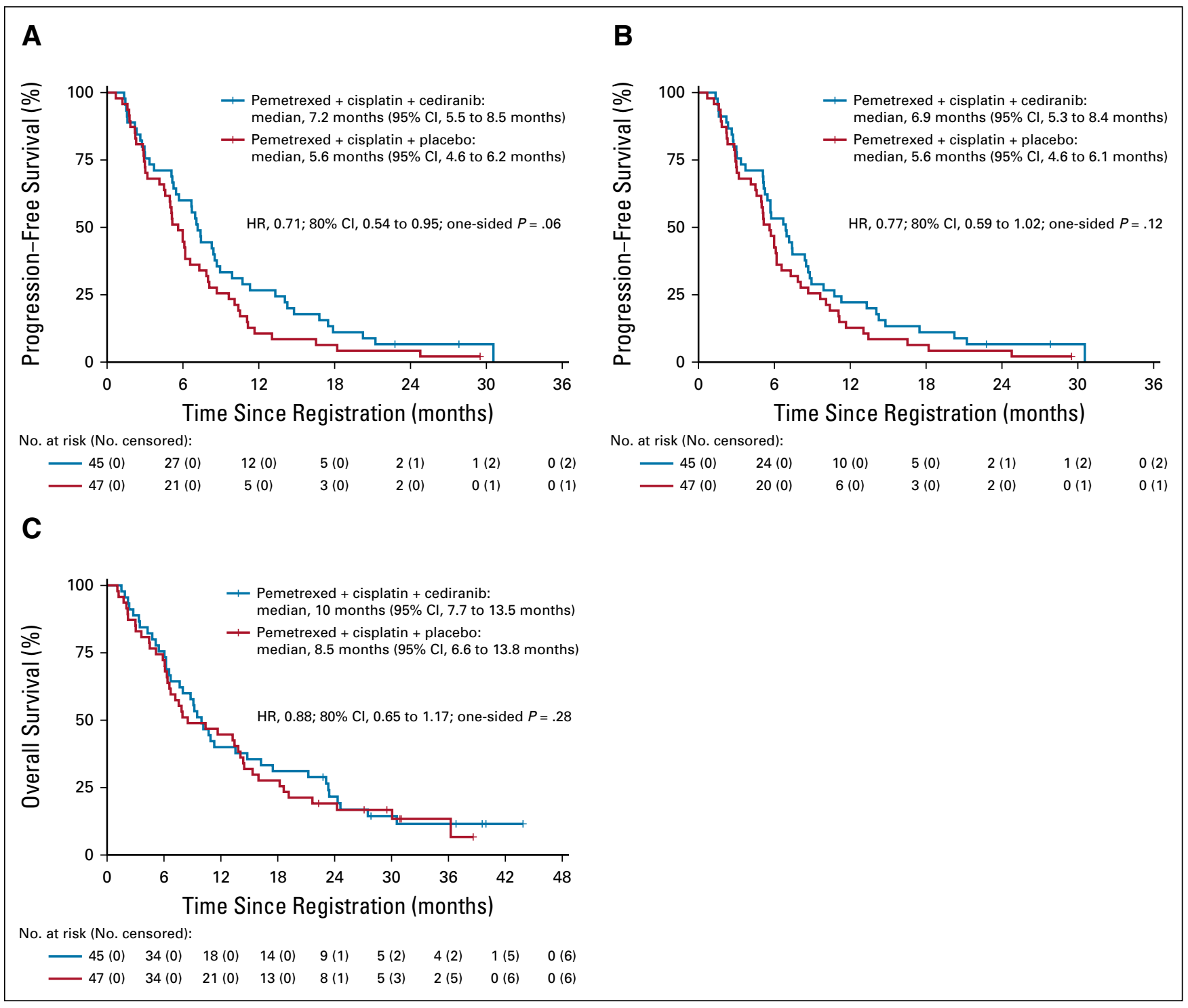

FIG 2. Progression-free survival outcomes by (A) RECIST v1.1 and (B) modified RECIST 1.1. (C) Overall survival curves. HR, hazard ratio.

and histology (epithelioid $v$ biphasic or sarcomatoid) using a dynamic balancing algorithm. ${ }^{15}$ Patients were enrolled via a Web-based application and simultaneous randomization was performed by a computer program. Patients and investigators were blinded to the treatment arm.

\section{Statistics}

The primary objective was comparing PFS by RECIST v1.1. The trial was designed with $83 \%$ power to rule out no difference in PFS by RECIST v1.1 (hazard ratio $[\mathrm{HR}]=1$ ) at the one-sided $10 \%$ level if the true HR were 0.60 . The design required 78 PFS events and tested the null using a stratified log-rank test with statistical significance designated as a $P$ value $<.10$. It was estimated that 96 eligible patients were needed to achieve the number of PFS events. An interim futility analysis was performed after $50 \%$ of the expected events in the control arm occurred. This futility analysis was based on testing the alternative hypothesis (HR, 1.66) at the one-sided 0.005 level using a modified log-rank test to account for testing non-null HRs. PFS, disease control rate, and duration of response were analyzed by RECIST v1.1 and by mRECIST. Duration of response was defined as the date of partial/complete response until the date of disease progression or death, with patients censored at the date of last contact if last known to be alive and progression free. We used the Kaplan-Meier method to estimate OS, PFS, and duration of response, whereas confidence intervals for median survival were estimated using the Brookmeyer-Crowley method. A stratified log-rank test compared survival distributions and a stratified $\chi^{2}$ test compared binary end points at the $10 \%$ level. Consistent with the design on the basis of the one-sided $10 \%$ level, two-sided $80 \% \mathrm{Cls}$ are reported. Exploratory evaluations for PFS (RECIST v1.1 and mRECIST) 
TABLE 2. Efficacy Analysis

Survival Outcome

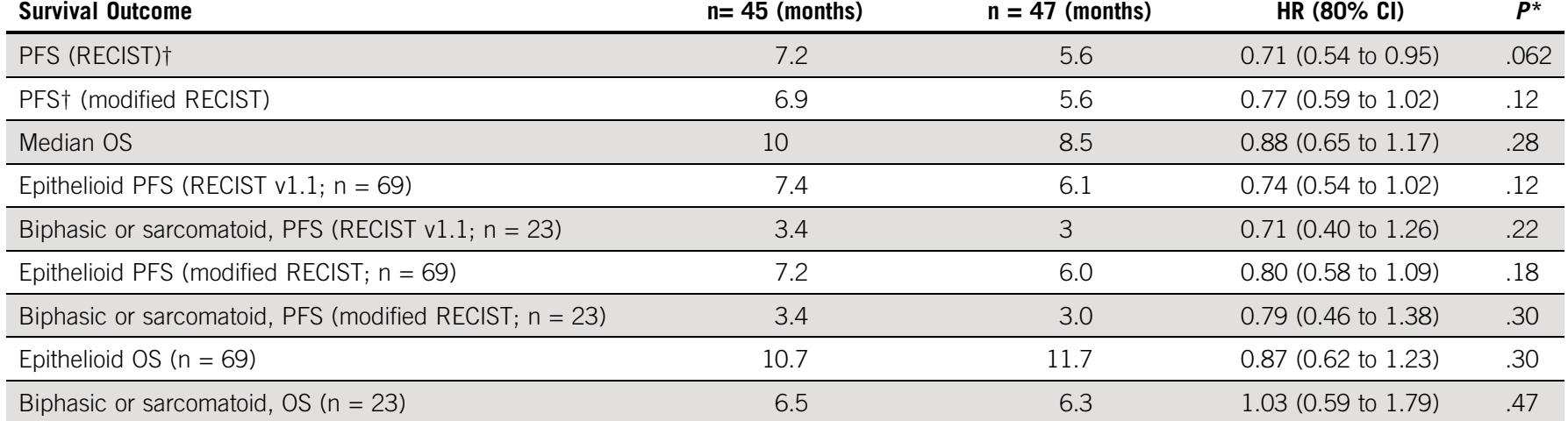

Abbreviations: HR, hazard ratio; OS, overall survival; PFS, progression-free survival.

*One-sided $P$ value with $80 \% \mathrm{Cl}$.

†Evaluable patients only.

and OS within subgroups defined by sex, prior chemotherapy, prior radiation therapy, prior surgery, performance, histology, and age were performed in separate models, including a first order and interaction term between the subgroup and treatment using Cox proportional hazards regression model. Adjustments for multiple comparisons were not performed. A 24-week landmark analysis postrandomization assessed the association between grade 3 and 4 hypertension or any grade 3 and 4 toxicity using Cox regression. HR and associated Cls were estimated using Cox proportional hazards regression model.

\section{RESULTS}

Between October 2011 and February 2016, 97 patients were enrolled, of whom 92 were found to be eligible. Reasons for ineligibility include two cases of inadequate renal function, one case of inadequate hematologic function, one case for which baseline scans were not performed within the protocol-specified timeframe, and one case where a second primary carcinoid tumor was found (Fig 1). Among eligible patients, median age was 72 years, with $85 \%$ of patients being men, $75 \%$ with epithelioid histology, and 25\% with biphasic or sarcomatoid histology (Table 1).

\section{Clinical Outcomes}

Median follow-up time was 31 months among patients who were still alive. Thirty-nine and 41 deaths have been reported in the cediranib and placebo arms, respectively. Two measurement systems-RECIST v1.1 and mRECIST - were performed on eligible patients for radiographic assessments by investigators. At baseline, 75 patients were measurable by RECIST v1.1 and 66 by mRECIST.

The trial met its primary objective with an improved RECIST PFS in the cediranib arm compared with the placebo arm (HR, 0.71; 80\% $\mathrm{Cl}, 0.54$ to $0.95 ; P=.062$; median PFS, 7.2 months $v 5.6$ months; Fig $2 \mathrm{~A}$ and Table 2). By mRECIST measurements, PFS was also improved in the cediranib arm but was not statistically significant (HR, $0.77 ; 80 \% \mathrm{Cl}, 0.59$ to $1.02 ; P=.12$; median, 6.9 months $v 5.6$ months). There was no significant difference in OS. Median OS was 10 months compared with 8.5 months in the placebo arm (HR, 0.88; $80 \% \mathrm{Cl}, 0.65$ to $1.17 ; P=.28$ ).

The cediranib arm had a statistically significantly higher mRECIST response rate compared with the placebo arm (50\% v20\%; $P=.006$ ). Duration of response numerically favored the cediranib arm (HR, $0.77 ; 80 \% \mathrm{Cl}, 0.41$ to 1.45 ; 4.1 months $v 3.7$ months; $P=.30$ ). By RECIST v1.1, the cediranib arm numerically improved the response rate (26\% v15\%; $P=.15)$ and duration of response (HR, 0.67; $80 \% \mathrm{Cl}, 0.31$ to $1.43 ; 6$ months $v 1.7$ months; $P=.25$ ) over that of the placebo. Figure 3 depicts the waterfall plots by RECIST v1.1 and MRECIST.

\section{Subgroup Analysis}

The following subgroup analyses were exploratory. Figure 4 describes the subgroup analysis by forest plot. Patients with epithelioid histology $(n=69)$ had better survival outcomes (Table 2) than did patients with biphasic and sarcomatoid histology $(n=23)$. This prognostic association was observed for OS favoring epithelioid patients (HR, 0.61; 80\% $\mathrm{Cl}, 0.44$ to $0.84 ; P=.024$; median, 10.9 months $\vee 6.4$ months). Median RECIST v1.1 PFS (HR, $0.62 ; 80 \% \mathrm{Cl}$, 0.45 to 0.85 ; $P=.026$; median, 7.1 months $v 3.4$ months) and mRECIST PFS (HR, $0.58 ; 80 \% \mathrm{Cl}, 0.42$ to $0.80 ; P=$ .014; median, 6.2 months $v 3.4$ months) were improved in patients with epithelioid histology compared with those with biphasic or sarcomatoid histology. However, there was no difference in treatment effect by histologic subtype (HR, $0.74 ; 80 \% \mathrm{Cl}, 0.54$ to 1.02 for epithelioid compared with 


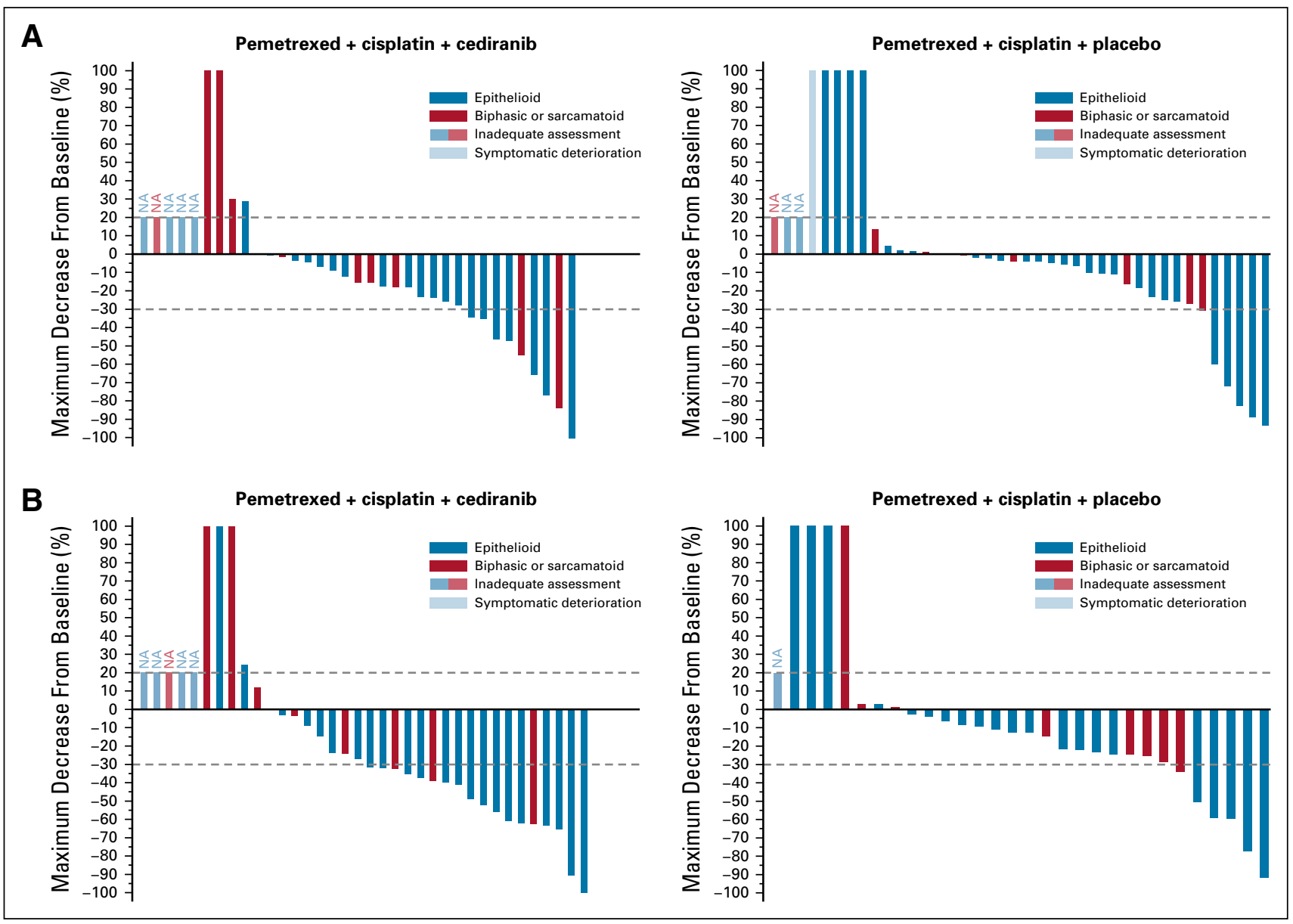

FIG 3. Waterfall plots by (A) RECIST v1.1 and (B) modified RECIST v1.1. Each vertical bar represents a patient's best percentage of decrease in tumor burden compared with baseline as defined by RECIST v1.1 or modified RECIST v1.1 measurements. Only patients with measurable disease at baseline are presented in these plots. Patients who did not have follow-up tumor disease assessment data are coded as inadequate assessment (NA). Patients who had new lesions appear at their first follow-up assessment or who died before the first scheduled disease assessment and whose death can reasonably be assumed to be a result of disease progression are represented graphically as a $100 \%$ increase in tumor burden. Patients who had symptomatic deterioration at first disease assessment are coded as symptomatic deterioration. (A) Per RECIST v1.1, in the cediranib arm, there were a total of 35 patients evaluable for response. In the placebo arm, there were a total of 40 patients evaluable for response. (B) Per modified RECIST, there were 36 patients in the cediranib arm evaluable for response. In the placebo arm, there were a total of 30 patients evaluable for response.

$\mathrm{HR}, 0.71 ; 80 \% \mathrm{Cl}, 0.40$ to 1.26 for biphasic or sarcomatoid histologies).

There is evidence to suggest that prior chemotherapy —-that is, neoadjuvant treatment before MPM recurrence-is a poor prognostic factor. There were six patients in each arm who received prior chemotherapy. Median OS was 6.7 months in previously treated patients compared with 10.4 months in those with no prior chemotherapy (HR, $1.8 ; 80 \%, 1.18$ to $2.74 ; P=.038$ ). Patients with prior chemotherapy exposure had worse response rates by RECIST v1.1. (0\% v23\%; $P=.091)$ and mRECIST (11\% v $40 \% ; P=.089$ ).

Although the subgroup analysis has small numbers, these analyses suggest possible differential effects within subgroups to the chemotherapy-cediranib combination. In the RECIST v1.1 PFS subgroup analysis (Fig 2A), male patients
(HR, 0.63; $P=.03$ ), patients with prior radiation therapy (HR, 0.32; $P=.03)$, any prior surgical procedure (HR, 0.55; $P=.04)$, and age 65 years or older (HR, 0.5; $P<.01)$ seemed to benefit from the addition of cediranib. The same subgroups seemed to benefit in the mRECIST PFS subgroup analyses (Fig 2B). It should be noted that the older patients (age $\geq 65$ years) had less prior chemotherapy as part of definitive treatment ( $9 \% \vee 25 \% ; P=.04)$. Likely because of small sample sizes and number of events, there were no clear subgroup associations for comparison of OS between arms (Fig 2C).

\section{Compliance and Toxicity}

Patients in the cediranib arm received fewer cycles of chemotherapy (median, four $v$ six; $P=.41$ ) and fewer days of cediranib/placebo use (median, 80 days $v 124$ days; $P=$ .31; Appendix Table A1, online only). More patients in the 


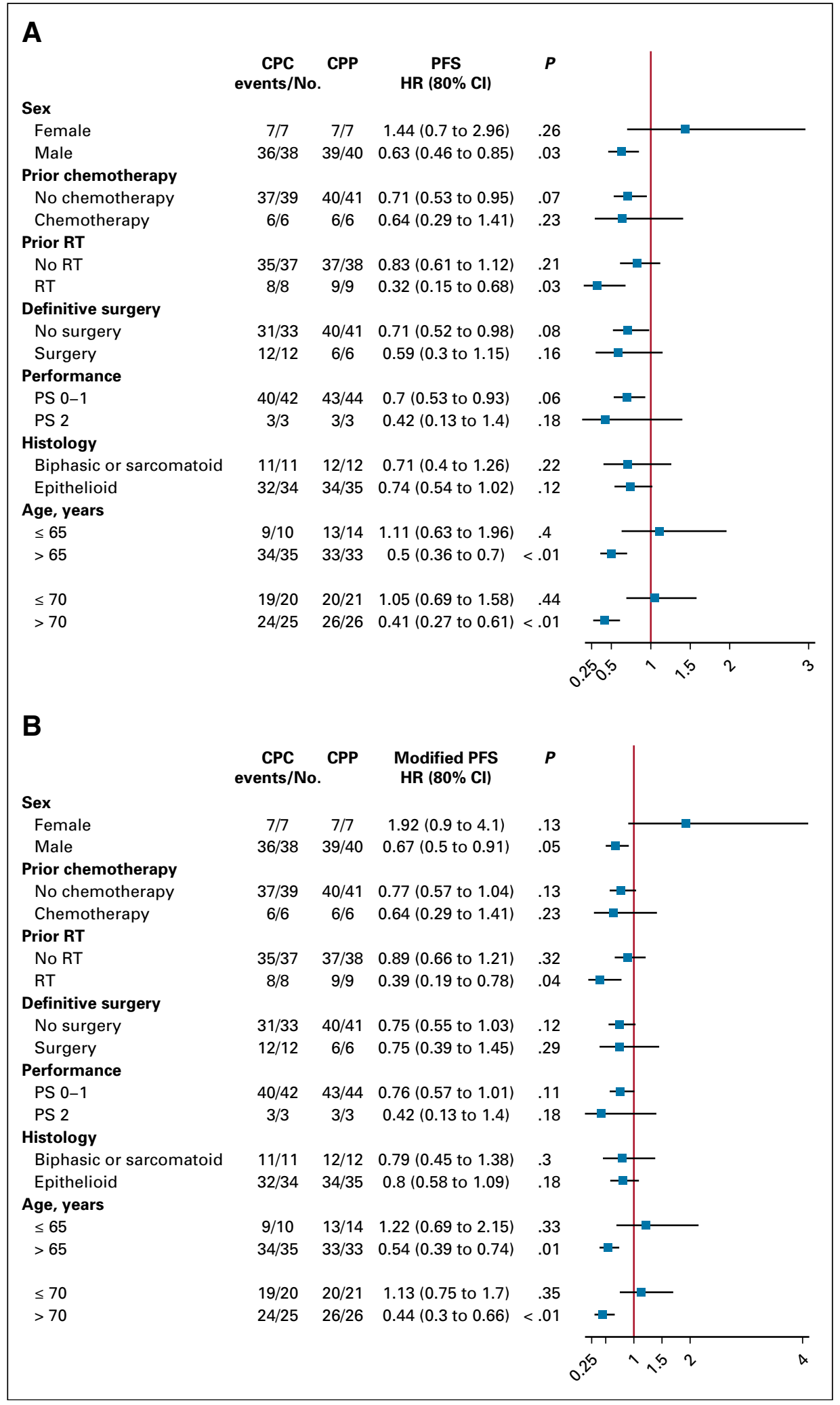

FIG 4. Forest plot subgroup analysis. (A) Progression-free survival (PFS) by RECIST 1.1, (B) PFS by modified RECIST, and (C) OS. CPC, carboplatin-pemetrexed-cediranib; CPP, carboplatin-pemetrexed-placebo; HR, hazard ratio; PS, performance status; RT, radiation therapy. cediranib arm switched from cisplatin to carboplatin (13\% v $4.3 \% ; P=.15)$, required a dose reduction of cediranib (13\% $\vee 2.1 \% ; P=.06)$, and discontinued cediranib as a result of toxicity ( $29 \% \vee 17 \% ; P=.18)$ compared with the placebo arm. Only $40 \%$ to $44 \%$ of patients from both arms were able to complete six cycles of platinumpemetrexed-cediranib/placebo and proceed to maintenance therapy. 


\begin{tabular}{|c|c|c|c|c|c|c|c|}
\hline & $\begin{array}{c}\text { CPC } \\
\text { events/No }\end{array}$ & CPP & $\begin{array}{c}\text { OS } \\
\text { HR }(80 \% \mathrm{Cl})\end{array}$ & $P$ & & & \\
\hline \multicolumn{8}{|l|}{ Sex } \\
\hline Female & $6 / 7$ & $5 / 7$ & $1.2(0.55$ to 2.62$)$ & .38 & & & \\
\hline Male & $33 / 38$ & $36 / 40$ & 0.84 (0.62 to 1.15$)$ & .24 & $\rightarrow-1$ & 十 & \\
\hline \multicolumn{8}{|l|}{ Prior chemotherapy } \\
\hline No chemotherapy & $33 / 39$ & $36 / 41$ & 0.88 (0.64 to 1.2$)$ & .3 & $\rightarrow+$ & F & \\
\hline Chemotherapy & $6 / 6$ & $5 / 6$ & 1.19 (0.54 to 2.63 ) & .39 & & 10 & \\
\hline \multicolumn{8}{|l|}{ Prior RT } \\
\hline No RT & $32 / 37$ & $33 / 38$ & 1.01 (0.74 to 1.39 ) & .48 & & & \\
\hline $\mathrm{RT}$ & $7 / 8$ & $8 / 9$ & 0.62 (0.31 to 1.25$)$ & .19 & & - & \\
\hline \multicolumn{8}{|l|}{ Definitive surgery } \\
\hline No surgery & $28 / 33$ & $36 / 41$ & 0.88 (0.64 to 1.22 ) & .31 & $\longrightarrow$ & - & \\
\hline Surgery & $11 / 12$ & $5 / 6$ & 0.75 (0.37 to 1.52$)$ & .3 & & & \\
\hline \multicolumn{8}{|l|}{ Performance } \\
\hline PS 0-1 & $36 / 42$ & $38 / 44$ & 0.91 (0.67 to 1.23 ) & .34 & & - & \\
\hline PS 2 & $3 / 3$ & $3 / 3$ & $0.42(0.13$ to 1.4$)$ & .18 & & & \\
\hline \multicolumn{8}{|l|}{ Histology } \\
\hline Biphasic/sarcomatoid & $11 / 11$ & $11 / 12$ & 1.03 (0.59 to 1.79 ) & .47 & & & \\
\hline Epitheliod/mesothelioma & $28 / 34$ & $30 / 35$ & 0.87 (0.62 to 1.23$)$ & .3 & & - & \\
\hline \multicolumn{8}{|l|}{ Age, years } \\
\hline$\leq 65$ & $8 / 10$ & $11 / 14$ & 1.47 (0.81 to 2.68 ) & .21 & & & \\
\hline$>65$ & $31 / 35$ & $30 / 33$ & $0.72(0.52$ to 1$)$ & .1 & - & & \\
\hline$\leq 70$ & $16 / 20$ & $17 / 21$ & 1.24 (0.79 to 1.95$)$ & .27 & & & \\
\hline \multirow[t]{2}{*}{$>70$} & $23 / 25$ & $24 / 26$ & 0.65 (0.44 to 0.95$)$ & .07 & -- & & \\
\hline & & & & & & פִ & 2 \\
\hline
\end{tabular}

FIG 4. (Continued).

The addition of cediranib to chemotherapy led to a higher rate of treatment-related adverse events compared with placebo (100\% v91\%; $P=.06$ ). Toxicities (any grade; Table 3) that were higher in the cediranib arm were anorexia ( $51 \%$ v38\%), diarrhea $(46.7 \% \vee 17 \%)$, epistaxis $(13 \% \vee 0 \%)$, muscle weakness (16\% v9\%), hypertension (44\% v 15\%), nausea (76\% v66\%), peripheral neuropathy (20\% v 11\%), weight loss (36\% $\vee 21 \%$ ), and myelosuppression (44\% $\vee 30 \%$ ). There were two cases of sinus bradycardia and one of supraventricular tachycardia in the cediranib arm compared with none in the placebo arm. The cediranib arm was associated with $69 \%$ grade 3 and 4 toxicities compared with $57 \%$ in the placebo arm $(P=.13)$. Four deaths occurred: two in the cediranib and one in the placebo arm from respiratory failure, and one sudden death in the placebo arm.

Grade 3 and 4 hypertension was not associated with worse prognosis. This was evaluated using a landmark analysis that evaluated the incidence of grade 3 and 4 hypertension within the six cycles of treatment and survival among patients who were alive at week 24 after random assignment. In this landmark analysis, there were 34 patients and 35 patients included from the cediranib and placebo arms, respectively. We similarly evaluated the association between any grade 3 and 4 toxicity and prognosis and showed no association ( $P=.32 ; \mathrm{HR}, 0.77)$.

\section{DISCUSSION}

Addition of cediranib to platinum-pemetrexed improved response rates (by mRECIST) and PFS (by RECIST v1.1) in patients with MPM. Although the sample size of the current study is small and patients were highly selected, there was a small clinical benefit with the addition of cediranib to cisplatin-pemetrexed. Numerically, the addition of cediranib led to a 1.3-month to 1.6-month improvement in median PFS and 1.5-month median OS over chemotherapy alone. It should be noted that the phase II SWOG S0905 underperformed in both arms for OS (median OS was 10 and 8.5 months in the cediranib and placebo arms, respectively) as the historical control for cisplatin-pemetrexed from the trial by Vogelzang et $\mathrm{al}^{1}$ had a median OS of 12.1 months and the MAPS trial ${ }^{7}$ bevacizumab arm had a median OS of 18.8 months and 16.1 months in the control arm. Why the phase II SWOG 0905 trial underperformed in both arms remains unclear, although patients with arguably poorer prognoses were enrolled $25 \%$ were biphasic/sarcomatoid, $20 \%$ had prior definitive surgery, and $13 \%$ had prior neoadjuvant chemotherapy). Neither the trial by Vogelzang et al, ${ }^{1}$ nor the MAPS trial ${ }^{7}$ included patients with prior exposure to neoadjuvant chemotherapy or extrapleural pneumonectomy. Thus, patients in SWOG S0905 may not be a comparative population given the higher proportion of patients with prior histories of aggressive therapy.

With the recent LUME-Meso phase III data, ${ }^{9}$ there is now consensus that multitargeted antiangiogenic tyrosine kinase inhibitors should not be administered with chemotherapy in unselected front-line MPM. The LUME-Meso phase || trial $^{8}$ originally reported much higher median OS 
TABLE 3. Adverse Events

\begin{tabular}{|c|c|c|c|c|c|c|c|c|}
\hline \multirow[b]{3}{*}{ Adverse Event } & \multicolumn{4}{|c|}{ Platinum + Pemetrexed + Cediranib $(n=45)$} & \multicolumn{4}{|c|}{ Platinum + Pemetrexed + Placebo $(n=47)$} \\
\hline & \multicolumn{4}{|c|}{ Grade } & \multicolumn{4}{|c|}{ Grade } \\
\hline & $1-2$ & 3 & 4 & 5 & $1-2$ & 3 & 4 & 5 \\
\hline Anemia & $24(53)$ & $1(2)$ & $0(0)$ & $0(0)$ & $21(45)$ & $6(13)$ & $1(2)$ & $0(0)$ \\
\hline Anorexia & $16(36)$ & $7(16)$ & $0(0)$ & $0(0)$ & $14(30)$ & $4(9)$ & $0(0)$ & $0(0)$ \\
\hline Atrial fibrillation/atrial flutter & $1(2)$ & $3(7)$ & $0(0)$ & $0(0)$ & $0(0)$ & $1(2)$ & $0(0)$ & $0(0)$ \\
\hline Creatinine increased & $12(27)$ & $0(0)$ & $0(0)$ & $0(0)$ & $12(26)$ & $1(2)$ & $0(0)$ & $0(0)$ \\
\hline Dehydration & $6(13)$ & $4(9)$ & $0(0)$ & $0(0)$ & $10(21)$ & $2(4)$ & $0(0)$ & $0(0)$ \\
\hline Diarrhea & $19(42)$ & $2(4)$ & $0(0)$ & $0(0)$ & $8(17)$ & $0(0)$ & $0(0)$ & $0(0)$ \\
\hline Epistaxis & $5(11)$ & $1(2)$ & $0(0)$ & $0(0)$ & $0(0)$ & $0(0)$ & $0(0)$ & $0(0)$ \\
\hline Fatigue & $24(53)$ & $6(13)$ & $0(0)$ & $0(0)$ & $27(57)$ & $6(13)$ & $0(0)$ & $0(0)$ \\
\hline Heart failure & $0(0)$ & $1(2)$ & $0(0)$ & $0(0)$ & $0(0)$ & $0(0)$ & $0(0)$ & $0(0)$ \\
\hline Hypertension & $11(24)$ & $9(20)$ & $0(0)$ & $0(0)$ & $7(15)$ & $0(0)$ & $0(0)$ & $0(0)$ \\
\hline Hypotension & $0(0)$ & $3(7)$ & $0(0)$ & $0(0)$ & $3(6)$ & $0(0)$ & $0(0)$ & $0(0)$ \\
\hline Intracranial hemorrhage & $0(0)$ & $0(0)$ & $1(2)$ & $0(0)$ & $0(0)$ & $0(0)$ & $0(0)$ & $0(0)$ \\
\hline Muscle weakness & $6(13)$ & $1(2)$ & $0(0)$ & $0(0)$ & $3(6)$ & $1(2)$ & $0(0)$ & $0(0)$ \\
\hline Myelosuppression & $17(38)$ & $1(2)$ & $2(4)$ & $0(0)$ & $10(21)$ & $3(6)$ & $1(2)$ & $0(0)$ \\
\hline Nausea & $30(67)$ & $4(9)$ & $0(0)$ & $0(0)$ & $26(55)$ & $5(11)$ & $0(0)$ & $0(0)$ \\
\hline Neutrophil count decreased & $11(24)$ & $5(11)$ & $1(2)$ & $0(0)$ & $8(17)$ & $5(11)$ & $4(9)$ & $0(0)$ \\
\hline Peripheral sensory neuropathy & $9(20)$ & $0(0)$ & $0(0)$ & $0(0)$ & $4(9)$ & $1(2)$ & $0(0)$ & $0(0)$ \\
\hline Platelet count decreased & $10(22)$ & $3(7)$ & $1(2)$ & $0(0)$ & $8(17)$ & $1(2)$ & $1(2)$ & $0(0)$ \\
\hline Rash maculopapular & $2(4)$ & $1(2)$ & $0(0)$ & $0(0)$ & $3(6)$ & $0(0)$ & $0(0)$ & $0(0)$ \\
\hline Sinus bradycardia & $0(0)$ & $2(4)$ & $0(0)$ & $0(0)$ & $0(0)$ & $0(0)$ & $0(0)$ & $0(0)$ \\
\hline Thromboembolic event & $2(4)$ & $0(0)$ & $3(7)$ & $0(0)$ & $2(4)$ & $1(2)$ & $2(4)$ & $0(0)$ \\
\hline Vomiting & 15 (33) & $0(0)$ & $0(0)$ & $0(0)$ & $13(28)$ & $2(4)$ & $0(0)$ & $0(0)$ \\
\hline Weight loss & 12 (27) & $4(9)$ & $0(0)$ & $0(0)$ & $10(21)$ & $0(0)$ & $0(0)$ & $0(0)$ \\
\hline
\end{tabular}

NOTE. Data presented as No. (\%).

results (20.6 months in nintedanib arm $v 15.2$ months in the chemotherapy alone arm), but the recent LUME-Meso phase III trial (epithelioid only) was a negative study (median PFS: 6.8 months nintedanib $v 7.0$ months placebo). ${ }^{9}$ At a median follow up of 9.2 months (nintedanib) and 9.7 months (placebo), median OS for the nintedanib arm was 14.4 months and 16.1 months in the placebo arm (HR, 1.12; $P=.538$ ). It remains unknown why the LUME-Meso phase II trial had a dramatic benefit that failed to translate to the phase III. It is therefore essential to identify whether there are specific populations of patients with MPM who can achieve a survival benefit with antiangiogenic therapies.

In SWOG 0905, the clinical benefit to cediranib was more pronounced in patients who typically have a worse prognosis, which includes men, patients with prior radiation therapy exposure, those with any prior surgical procedure, PS of 2 , and those older than age 65 years. It is unusual that women had worse outcomes compared with men. This directly contradicts known mesothelioma data that indicate that women have improved OS compared with men and respond better to chemotherapy; in the MAPS trial, ${ }^{7}$ women had an HR of 0.70 that favored the bevacizumab arm. Our findings may be consistent with the E4599 non-small-cell lung cancer data that show that women achieve less benefit with antiangiogenic therapy. ${ }^{16}$

One can hypothesize that radiation therapy and surgery may induce proangiogenic factors and that targeting VEGFR/PDGFR after may improve the antitumor effect. The benefit to older patients and patients with a PS of 2 is less clear; however, this may be a result, in part, of the confounding factor that younger patients had more prior chemotherapy use. It is also possible that patients with a PS of 2 had a poor PS because of their disease and that a greater debulking effect may have improved survival.

The triplet regimen with cediranib was associated with GI, hypertension, weight loss, epistaxis, and rarely with cardiac toxicities. Compared with placebo, patients receiving cediranib were not able to complete as many cycles of the triplet regimen, 29\% discontinued cediranib as a result of 
toxicity, and $13 \%$ required dose reductions. More grade 3 and higher toxicities occurred with cediranib (69\%) compared with placebo (57\%). Compared with the MAPS trial, ${ }^{2}$ the toxicity of the cisplatin-pemetrexed-cediranib regimen was similar to that of the cisplatin-pemetrexed-bevacizumab regimen, which reported a $71 \%$ grade 3 and 4 adverse event rate.

Although our phase I SWOG S0905 triall ${ }^{12}$ reported a low grade 3 hypertension incidence, this phase II study demonstrated a $20 \%$ grade 3 hypertension rate with cediranib, which is comparable to that of the MAPS trial (23\%). Similar to the MAPS trial in which a $6 \%$ grade 3 thromboembolic rate was observed, cisplatin-pemetrexed-cediranib had a $7 \%$ grade 3 rate. There was one report of grade 3 intracranial hemorrhage and six cases of epistaxis (five grade 1 cases) in the cediranib arm (16\%), whereas MAPS reported a $37.4 \%$ rate of hemorrhage (primarily grade 1 and 2 epistaxis). There was no correlation of survival benefit in patients who experienced hypertension or any other adverse event.

On the basis of the minor survival improvement and toxicity profile, cediranib will not be developed further for mesothelioma. To date, the French Cooperative Thoracic Intergroup MAPS trial ${ }^{7}$ with bevacizumab remains the only positive phase III trial with an antiangiogenic in a large number of patients with MPM. This triplet regimen is included in the National Comprehensive Cancer Network guidelines as an option for front-line therapy in patients with MPM. Overall, antiangiogenics seem to provide clinical benefit for a subgroup of patients with MPM. It is essential that future studies include translational correlates for predictive biomarkers that can identify this population of patients. There is clear evidence that the addition of cediranib increased response rates and reduced tumor

\author{
AFFILIATIONS \\ ${ }^{1}$ The University of Texas MD Anderson Cancer Center, Houston, TX \\ ${ }^{2}$ SWOG Statistical Center, Seattle, WA \\ ${ }^{3}$ US Oncology Comprehensive Cancer Centers of Nevada, Las Vegas, NV \\ ${ }^{4}$ Cancer Care Specialists of Illinois, Decatur, IL \\ ${ }^{5}$ Kaiser Permanente, Fresno, CA \\ ${ }^{6}$ Montana Cancer Consortium, Billings, MT \\ ${ }^{7}$ University of Michigan, Ann Arbor, MI \\ ${ }^{8}$ University of California Davis Cancer Center, Sacramento, CA
}

\section{CORRESPONDING AUTHOR}

Anne S. Tsao, MD, Department of Thoracic and Head and Neck Medical Oncology, Unit 432, The University of Texas MD Anderson Cancer Center, 1515 Holcombe Blvd, Houston, TX 77030; e-mail: astsao@ mdanderson.org.

\section{SUPPORT}

Supported by National Cancer Institute Grants No. CA180888, CA180819, CA180858, CA180801, CA189830, CA189821, CA189872, CA180830 CA180835, CA189858, CA189971, CA189972, and CA180846. burden; however, this did not translate into a significant OS improvement. Future therapeutic strategies should consider improving on maintenance therapy strategies to prolong the duration of response and ideally be defined by predictive biomarkers. A major limitation of our trial was the lack of biomarker analysis.

There is scientific rationale to combine immunotherapies with antiangiogenics. Preclinical studies in other solid tumors have shown interactions between T-cell activation and angiogenesis and suggest that dual inhibition can be synergistic. ${ }^{17,18}$ Lucchi et al ${ }^{19}$ determined that in interleukin2-treated patients with mesothelioma, cytotoxic CD8 ${ }^{+}$ lymphocytes and tryptase mast cells increased and microvessel number and tumor angiogenesis decreased. In front-line non-small-cell lung cancer, the IMPower150 (ClinicalTrials.gov identifier: NCT02366143) trial$^{20}$ improved OS with the quadruplet carboplatin-paclitaxelbevacizumab-atezolizumab compared with carboplatinpaclitaxel-bevacizumab (19.2 months $v 14.7$ months; HR for death, $0.78 ; 95 \% \mathrm{Cl}, 0.64$ to $0.96 ; P=.02$ ). Two studies are ongoing in salvage mesothelioma, evaluating the combinations of bevacizumab and atezolizumab (ClinicalTrials.gov identifier: NCT03074513) and pembrolizumab and nintedanib (ClinicalTrials.gov identifier: NCT02856425).

In conclusion, addition of cediranib to platinumpemetrexed improves response rates (mRECIST) and PFS (RECIST v1.1) in patients with MPM. However, in light of the toxicity profile, the incremental survival benefit is not sufficient to justify additional development of cediranib in mesothelioma. Future strategies should focus on identifying predictive biomarkers and consider combining antiangiogenics with immunotherapies to enhance the magnitude and duration of response.

AUTHORS' DISCLOSURES OF POTENTIAL CONFLICTS OF INTEREST AND DATA AVAILABILITY STATEMENT

Disclosures provided by the authors and data availability statement (if applicable) are available with this article at DOI https://doi.org/10.1200/ JC0.19.00269

\section{AUTHOR CONTRIBUTIONS}

Conception and design: Anne S. Tsao, Nicholas J. Vogelzang, Frank V. Fossella, Mary W. Redman, David R. Gandara

Administrative support: Anne S. Tsao

Provision of study materials or patients: Anne S. Tsao, John V. Heymach, Frank V. Fossella, Charles Lu, Mario R. Velasco, Brandy Box-Noriega, James G. Hueftle, Shirish Gadgeel, David R. Gandara

Collection and assembly of data: Anne S. Tsao, Ignacio I. Wistuba,

Nicholas J. Vogelzang, Frank V. Fossella, Mario R. Velasco, Brandy BoxNoriega, James G. Hueftle, Shirish Gadgeel, Mary W. Redman

Data analysis and interpretation: Anne S. Tsao, Jieling Miao, Nicholas

J. Vogelzang, John V. Heymach, Frank V. Fossella, Charles Lu, Shirish Gadgeel, Mary W. Redman, David R. Gandara, Karen Kelly

Manuscript writing: All authors

Final approval of manuscript: All authors

Accountable for all aspects of the work: All authors 


\section{REFERENCES}

1. Vogelzang NJ, Rusthoven JJ, Symanowski J, et al: Phase III study of pemetrexed in combination with cisplatin versus cisplatin alone in patients with malignant pleural mesothelioma. J Clin Oncol 21:2636-2644, 2003

2. Zebrowski BK, Yano S, Liu W, et al: Vascular endothelial growth factor levels and induction of permeability in malignant pleural effusions. Clin Cancer Res 5: 3364-3368, 1999

3. Strizzi L, Catalano A, Vianale G, et al: Vascular endothelial growth factor is an autocrine growth factor in human malignant mesothelioma. J Pathol 193:468-475, 2001

4. Ohta Y, Shridhar V, Bright RK, et al: VEGF and VEGF type C play an important role in angiogenesis and lymphangiogenesis in human malignant mesothelioma tumours. Br J Cancer 81:54-61, 1999

5. Uehara H, Kim SJ, Karashima T, et al: Effects of blocking platelet-derived growth factor-receptor signaling in a mouse model of experimental prostate cancer bone metastases. J Natl Cancer Inst 95:458-470, 2003

6. Pietras K, Ostman A, Sjöquist M, et al: Inhibition of platelet-derived growth factor receptors reduces interstitial hypertension and increases transcapillary transport in tumors. Cancer Res 61:2929-2934, 2001

7. Zalcman G, Mazieres J, Margery J, et al: Bevacizumab for newly diagnosed pleural mesothelioma in the Mesothelioma Avastin Cisplatin Pemetrexed Study (MAPS): A randomised, controlled, open-label, phase 3 trial. Lancet 387:1405-1414, 2016

8. Grosso F, Steele N, Novello S, et al: Nintedanib plus pemetrexed/cisplatin in patients with malignant pleural mesothelioma: Phase II results from the randomized, placebo-controlled LUME-Meso trial. J Clin Oncol 35:3591-3600, 2017

9. Scagliotti G, Gaafar R, Nowak A, et al: Nintedanib + Pemetrexed/Cisplatin in Patients With Unresectable MPM: Phase III Results From the LUME-Meso Trial. Toronto, ON, Canada, International Association for the Study of Lung Cancer, 2018

10. Garland LL, Chansky K, Wozniak AJ, et al: Phase II study of cediranib in patients with malignant pleural mesothelioma: SWOG S0509. J Thorac Oncol 6: 1938-1945, 2011

11. Erber R, Thurnher A, Katsen AD, et al: Combined inhibition of VEGF and PDGF signaling enforces tumor vessel regression by interfering with pericyte-mediated endothelial cell survival mechanisms. FASEB J 18:338-340, 2004

12. Tsao AS, Moon J, Wistuba II, et al: Phase I trial of cediranib in combination with cisplatin and pemetrexed in chemonaive patients with unresectable malignant pleural mesothelioma (SWOG S0905). J Thorac Oncol 12:1299-1308, 2017

13. Byrne MJ, Nowak AK: Modified RECIST criteria for assessment of response in malignant pleural mesothelioma. Ann Oncol 15:257-260, 2004

14. Tsao AS, Garland L, Redman M, et al: A practical guide of the Southwest Oncology Group to measure malignant pleural mesothelioma tumors by RECIST and modified RECIST criteria. J Thorac Oncol 6:598-601, 2011

15. Pocock SJ, Simon R: Sequential treatment assignment with balancing for prognostic factors in the controlled clinical trial. Biometrics $31: 103-115$, 1975

16. Brahmer JR, Dahlberg SE, Gray RJ, et al: Sex differences in outcome with bevacizumab therapy: Analysis of patients with advanced-stage non-small cell lung cancer treated with or without bevacizumab in combination with paclitaxel and carboplatin in the Eastern Cooperative Oncology Group Trial 4599. J Thorac Oncol 6:103-108, 2011

17. Kaur S, Chang T, Singh SP, et al: CD47 signaling regulates the immunosuppressive activity of VEGF in T cells. J Immunol 193:3914-3924, 2014

18. Hilberg F, Reschke M, Hofmann M, et al: Nintedanib improves anti-tumor efficacy in combination with anti PD-1 in syngeneic tumor models sensitive and refractory to 10 inhibition. J Thorac Oncol 12:S813, 2017 (suppl)

19. Lucchi M, Picchi A, Ali G, et al: Multimodality treatment of malignant pleural mesothelioma with or without immunotherapy: Does it change anything? Interact Cardiovasc Thorac Surg 10:572-576, 2010

20. Socinski MA, Jotte RM, Cappuzzo F, et al: Atezolizumab for first-line treatment of metastatic nonsquamous NSCLC. N Engl J Med 378:2288-2301, 2018

\section{Cancer.Net Mobile App for Patients}

Cancer.Net's award-winning app is the mobile companion for patients to stay informed about cancer and to organize important personal data often needed for visits to physicians. It includes interactive tools to help patients get answers to important questions, track side effects, and manage medications. Patients using Spanish language - enabled devices can also access the tools and information in Spanish. Direct your patients to cancer.net/app to download the Cancer.Net mobile app. 
Phase II Trial of Cediranib in Combination With Cisplatin and Pemetrexed in Chemotherapy-Naïve Patients With Unresectable Malignant Pleural Mesothelioma (SWOG S0905)

The following represents disclosure information provided by authors of this manuscript. All relationships are considered compensated. Relationships are self-held unless noted. I = Immediate Family Member, Inst = My Institution. Relationships may not relate to the subject matter of this manuscript. For more information about ASCO's conflict of interest policy, please refer to www.asco.org/rwc or ascopubs.org/jco/site/ifc.

Anne S. Tsao

Consulting or Advisory Role: Novartis, Boehringer Ingelheim, Genentech, Medlmmune, Imedex, Eli Lilly, Bristol-Myers Squibb, Epizyme, AstraZeneca, Medlmmune, Ariad Pharmaceuticals, EMD Serono, Takeda, Heron Research Funding: Medlmmune, Merck, Genentech, Seattle Genetics, Millennium Pharmaceuticals, Bristol-Myers Squibb, Boehringer Ingelheim, Polaris, Bristol-Myers Squibb, EMD Serono, Seattle Genetics Patents, Royalties, Other Intellectual Property: UpToDate

\section{Ignacio I. Wistuba}

Consulting or Advisory Role: Genentech, Bristol-Myers Squibb, HTG Molecular Diagnostics, Asuragen, Pfizer, AstraZeneca, Medlmmune, GlaxoSmithKline, Guardant Health

Speakers' Bureau: Pfizer, MSD Oncology, Bristol-Myers Squibb, Roche Research Funding: Genentech (Inst), Merck (Inst), HTG Molecular Diagnostics (Inst), Silicon Biosystems (Inst), Adaptimmune (Inst), EMD Serono (Inst), Pfizer (Inst), Medlmmune (Inst), OncoPlex Diagnostics (Inst), Takeda (Inst), Karus Therapeutics (Inst), Amgen (Inst), 4D Molecular Therapeutics (Inst), Bayer (Inst), Novartis (Inst), Guardant Health (Inst)

Travel, Accommodations, Expenses: Genentech, Bristol-Myers Squibb, HTG Molecular Diagnostics, Pfizer, AstraZeneca, Medlmmune, GlaxoSmithKline, Guardant Health, MSD Oncology

Nicholas J. Vogelzang

Employment: US Oncology

Stock and Other Ownership Interests: Caris Life Sciences

Honoraria: UpToDate, Pfizer, Novartis

Consulting or Advisory Role: Pfizer, Bayer, Genentech, Heron, AstraZeneca, Caris Life Sciences, Fujifilm, Tolero Pharmaceuticals, Merck, Astellas Pharma, Boehringer Ingelheim

Speakers' Bureau: Bayer, Sanofi, Genentech, Bristol-Myers Squibb, Exelixis, AstraZeneca, Medlmmune

Research Funding: US Oncology (Inst), Endocyte (Inst), Merck (Inst), Suzhou Kintor Pharmaceuticals (Inst)

Expert Testimony: Novartis

Travel, Accommodations, Expenses: Genentech, US Oncology, Pfizer, Bayer, Onyx Pharmaceuticals, Exelixis, AstraZeneca, Medlmmune, Sanofi

John V. Heymach

Stock and Other Ownership Interests: Cardinal Spine, Bio-Tree

Consulting or Advisory Role: AstraZeneca, Boehringer Ingelheim, Bristol-Myers Squibb, Spectrum Pharmaceuticals, Novartis, Genentech, Guardant Health, Hengrui Pharmaceutical, GlaxoSmithKline, EMD Serono, Merck, Eli Lilly, Syntha Pharmaceutical, Takeda, Sanofi

Research Funding: AstraZeneca (Inst), Spectrum Pharmaceuticals, Bayer, GlaxoSmithKline

Patents, Royalties, Other Intellectual Property: Licensing agreement between Spectrum and MD Anderson, including myself, regarding intellectual property for treatment of EGFR and HER2 exon 20 mutations

\section{Frank V. Fossella}

Honoraria: NovoCure, Takeda Science Foundation, EMD Serono

Consulting or Advisory Role: EMD Serono, Takeda, Novocure

Travel, Accommodations, Expenses: EMD Serono, Takeda, Novocure

Mario R. Velasco

Employment: Cancer Care Specialists of Illinois

James G. Hueftle

Stock and Other Ownership Interests: Johnson \& Johnson, Amgen, BristolMyers Squibb, Pfizer, Merck, Eli Lilly

\section{Shirish Gadgeel}

Honoraria: Genentech, AstraZeneca

Consulting or Advisory Role: Pfizer, Genentech, AstraZeneca, Bristol-Myers

Squibb, AbbVie, Takeda, Xcovery, Boehringer Ingelheim

Research Funding: Pfizer (Inst), Merck, Genentech (Inst), Merck (Inst),

Blueprint Medicines (Inst), Ariad Pharmaceuticals (Inst), Takeda (Inst), Aeglea Biotherapeutics (Inst), Astellas Pharma (Inst), G1 Therapeutics (Inst), Lycera (Inst), Daiichi Sankyo (Inst)

Travel, Accommodations, Expenses: Genentech, AstraZeneca

Other Relationship: AstraZeneca

Mary W. Redman

Consulting or Advisory Role: Ignyta

David R. Gandara

Consulting or Advisory Role: Celgene, AstraZeneca, Genentech, Eli Lilly, Guardant Health, CellMax Life, Pfizer

Research Funding: Genentech (Inst), AstraZeneca (Inst), MedImmune (Inst)

\section{Karen Kelly}

Honoraria: Merck

Consulting or Advisory Role: AstraZeneca, Genentech, Eli Lilly, Regeneron, AbbVie, Janssen, EMD Serono, Merck, Pfizer, AstraZeneca

Research Funding: EMD Serono (Inst), Genentech (Inst), AbbVie (Inst), Five Prime Therapeutics (Inst), Regeneron (Inst)

Patents, Royalties, Other Intellectual Property: Author royalties for UpToDate, an evidence-based, peer-reviewed information resource, available via the Web, desktop, and PDA

Travel, Accommodations, Expenses: AstraZeneca, Genentech, Merck, Regeneron, Eli Lilly, AbbVie, EMD Serono, Merck

No other potential conflicts of interest were reported. 


\section{APPENDIX}

TABLE A1. Compliance With Therapy

Compliance

Median No. of chemotherapy cycles

Median No. of days of cediranib or placebo use

Median total No. of days on therapy (from day 1 start of drug)

Completed six cycles and received maintenance cediranib or placebo, \%

Required dose reduction of cisplatin $75 \mathrm{mg} / \mathrm{m}^{2}$ to $60 \mathrm{mg} / \mathrm{m}^{2}$, \%

Patients who switched from cisplatin to carboplatin, \%

Required dose reduction of cediranib or placebo, \%

Discontinued cediranib or placebo as a result of toxicity, \%

Treatment-related death, \%
Platinum + Pemetrexed + Cediranib $(n=45)$

*One-sided $P$ value with $80 \% \mathrm{Cl}$.
Platinum + Pemetrexed + Placebo $(n=47)$

.41

$\begin{array}{rrr}4 & 6 & .41 \\ 80 & 124 & .31 \\ 97 & 123 & .80\end{array}$

44 (20 of 45$)$

40 (19 of 47)

.70

4.4 (2 of 45$)$

11 ( 5 of 47 )

.44

13 ( 6 of 45$)$

4.3 (2 of 47) .15

13 (6 of 45$)$

2.1 (1 of 47)

.06

29 (13 of 45)

17 (8 of 47)

.18

4.4 (2 of 45 )

4.3 (2 of 47 )

1.0

